L'ouvrage se termine par une note sur l'édition de Pierre Coste (La Haye, 1727) et une copieuse bibliographie sur les manuscrits, les principales éditions, les traductions et la réception critique de 1553 à nos jours. Un Index permet de repérer rapidement les noms propres et les principaux opuscules de référence.

FRANÇOIS RIGOLOT, Princeton University

\title{
Stéphanie Lecompte.
}

La Chaîne d'or des poètes. Présence de Macrobe dans l'Europe humaniste. Genève : Droz, Travaux d'Humanisme et Renaissance 449, 2009, 485 p.

Dans le chapitre III, 13, des Essais, « De l'Expérience », après un développement sur les usages divers des nations, Montaigne, à la recherche de la vérité, refuse de privilégier la tradition livresque sur le témoignage oral du présent; il dénonce « la sottise qui nous fait courir après les exemples estrangers et scholastiques » :

J'allègue aussi volontiers un mien amy, que Aulugelle, et que Macrobe : et ce que j'ay veu, que ce qu'ils ont escrit.

(Les Essais, III, 13 [1595], Paris : Gallimard, Bibliothèque de la Pléiade, 2007, p. 1129.)

Non que les auteurs antiques n'aient plus rien à nous dire ; leur discours reste chargé de sens et demeure fécond à qui sait l'entendre. La critique de Montaigne porte en fait sur l'usage pédant de «l'honneur de l'allégation » et non pas sur les auteurs allégués. Accessoirement, à travers les deux exemples qu'il cite dans son argumentation, il évoque deux auteurs latins, sources, sinon modèles, de son propre livre. La présence d'Aulu Gelle, à qui il emprunte diverses anecdotes et la conception générale d'une composition fondée sur l'ordo fortuitus, a fait l'objet d'une étude précise. Macrobe en revanche a été négligé par la critique ; il n’est pas retenu par Pierre Villey dans Les Sources et l'Evolution des Essais, il ne fait pas l'objet d'une notice dans le Dictionnaire de Michel de Montaigne et son nom n’apparaît pas dans l'ouvrage de référence de Hugo Friedrich, pourtant si attentif à la tradition lettrée qui nourrit les Essais. Or Montaigne ne cite pas l'auteur des Saturnales et du commentaire sur le Songe de Scipion comme un simple nom, vide de sens, «fausse fenêtre » dans un simple balancement oratoire, ni comme 
le second terme d'un couple topique, référence à l'édition réunissant les deux auteurs procurée par Henri Estienne, publiée en 1585 seulement. Même réduite à une seule citation dans le chapitre I, 18, à laquelle il faut ajouter deux allusions au Songe de Scipion, la présence de Macrobe est attestée dans les Essais, dès la première rédaction du livre. Elle ressortit peut-être à des souvenirs scolaires, elle correspond plus certainement encore aux modes mêmes de composition des Essais, fondés sur des lectures toujours renouvelées et, du moins dans une première phase, sur l'utilisation d'encyclopédies, de recueils d'apophtegmes et de compilations, comme à leur style combinant la pratique savante des excerpta et une forme de conversation mondaine en langue vernaculaire.

Si les études consacrées à Montaigne ignorent Macrobe, le livre de Stéphanie Lecompte, traitant de la présence de Macrobe dans l'Europe humaniste ne mentionne pas davantage Montaigne, en dépit de la citation des Essais, qui constitue pourtant un témoignage de la présence et de la fortune (ou l'infortune) de l'auteur latin tard dans le $\mathrm{XVI}^{\mathrm{e}}$ siècle et dans la culture des élites nobiliaires. Ce silence ou cet oubli peuvent se justifier. Ils illustrent à leur manière l'état des études montaignistes et non pas une lacune dans un livre reposant sur une documentation assez complète (même si, au demeurant, il y manque un inventaire des mentions à Macrobe). De surcroît, Montaigne n'était pas, stricto sensu, un « humaniste », et n'avait donc pas, en tant que tel, à être pris en considération. En fait, la relation de Montaigne à Macrobe, négligée jusqu’alors, prend pour nous un sens plus riche après la lecture attentive du livre de Mme Lecompte. C' est là sans doute un des mérites de cette étude, qui, en dépit d'une argumentation répétitive faute d'un bon plan, de paraphrases trop longues et d'un usage maladroit de certaines notions rhétoriques (ainsi "topos", toujours vide de sens), ouvre des perspectives inédites à ce jour sur la littérature française, en prolongeant et en complétant pour cette période les stimulantes suggestions que Rober Curtius avait formulées pour le Moyen Âge et la romania dans son ensemble. Certes, on cherchera en vain chez Macrobe de nouvelles « sources » et l'origine d'anecdotes mentionnées dans les Essais, même si cette enquête demanderait à être reprise sur de nouveaux frais. En revanche, on gagnera assurément à relire à la lumière des Saturnales et de leur commentaire de l'Enéide ce que Montaigne avait écrit de la poésie en général, et de Virgile en particulier ; on comprendra alors de façon plus précise la portée des catégories stylistiques qu’il emploie et leur lien avec la conception enthousiaste qu'il proposait de la poésie. 
Si le personnage reste toujours mystérieux, l’œuvre de Macrobe était bien connue à la Renaissance et ne cessa d'être lue des savants, comme elle l'avait été au Moyen Âge et pour les mêmes raisons. Le livre imprimé a simplement mis en forme une abondante tradition textuelle connue par plus de 600 manuscrits, en réunissant définitivement des œuvres jusqu’alors séparées. Cette diffusion toutefois avait une géographie qui se transforma au cours du XVI ${ }^{\mathrm{e}}$ siècle : d’abord italienne, en relation aux cercles néoplatoniciens de Florence et aux imprimeurs vénitiens, elle s'élargit à l'Europe entière, suivant dans ce cas particulier un mouvement plus général des études littéraires et de la librairie. Après cinq éditions incunables (l'editio princeps étant celle imprimée à Venise en 1472 par Nicolas Janson), on compte 28 éditions publiées au XVI ${ }^{\mathrm{e}}$ siècle (en fait un dizaine d'éditions véritables, auxquelles s'ajoutent des réimpressions voire de simples émissions sous des dates différentes), procurées par des savants de toute l'Europe : Rivius (1513), Haldrein (1521), Camerarius (1535), Estienne (1585), et surtout Isaac Pontanus (1597), qui donna pour deux siècles un texte fautif établi sur un mauvais manuscrit. On ajoutera à ces noms celui de Rabelais, à qui l'on attribue désormais l'édition publiée en 1538 à Lyon, chez Sébastien Gryphe. L'intérêt pour l'œuvre de Macrobe était en premier lieu d’ordre documentaire, aux sources mêmes de l'ambition humaniste de la connaissance du monde antique et de sa restitutio par les antiquaires et les philologues. Ce n'est pas par hasard si Rabelais introduisait Macrobe comme un personnage de fiction dans le Quart Livre pour faire de lui un guide conduisant ses hôtes parmi les monuments antiques dont il leur déchiffrait les inscriptions. Macrobe était reçu comme un historien et un grammairien, dont le principal mérite était d'ordre doxologique, celui de conserver les fragments d'auteurs perdus. Cet intérêt que lui témoignaient les humanistes, et au premier chef Érasme, ne se bornait pas toutefois à cette dimension étroitement documentaire. Son commentaire au Songe de Scipion, conjuguant la leçon de Platon et de Porphyre, ne cessa d'être considéré comme un grand texte de philosophie et de cosmologie, autant qu'il faisait de lui une référence majeure dans l'interprétation allégorique des textes littéraires. Macrobe avait donné la première méthode pour analyser la mythologie des Anciens et formulé de façon aussi détaillée que précise une théorie de la fable, dont les Saturnales marquent la mise à l'épreuve appliquée à l'œuvre de Virgile. Cette réputation savante faisait de Macrobe un auteur, au sens précis du terme, en dépit d'une latinité peu élégante. Ce statut lui fut reconnu jusquà la rupture, au milieu du XVII ${ }^{e}$ siècle, entre l'érudition et la littérature, qui eut pour 
conséquence de rendre incompréhensible en termes de genre et de statut une œuvre aussi complexe telle que les Saturnales.

En analysant les mythes, Macrobe contribua plus que tout autre à la survivance des dieux antiques, dont il donna le répertoire détaillé et l'explication de leurs attributs. Il mit en forme un savoir que les mythographes de la Renaissance ne cessèrent de reprendre à leur compte et de commenter, de Boccace à Cartari, en passant par Goffroy Tory, qui dans son Champfleury l'allègue précisément comme une autorité. Ces mythes ainsi éclairés jouèrent un rôle déterminant dans la constitution des arts de mémoire, étudiés par France Yates; ils donnèrent aux arts visuels une de leurs ressources principales et la clef la plus stimulante de leur interprétation. Macrobe d'autre part moralisa les mythes antiques en les sélectionnant; il en fit des fables à caractère philosophique, ouvrant un chemin de connaissance vers une vérité de nature divine. Par son syncrétisme, il offrait la possibilité de leur réinterprétation postérieure en termes chrétiens. Sa conception de la narratio fabulosa et du mythe reposait sur un ensemble de notions dont, comme le fait Mme Lecompte, on peut suivre la trace sur la longue durée dans la reprise de marques lexicales précises, dans l'humanisme italien, de Boccace, à Landino, mais aussi chez Caelius Rhodiginus, qui, sans jamais citer Macrobe nommément, lui emprunte l'essentiel de ses analyses de l'allégorie, puis dans l'humanisme français, autour de Josse Bade, avant sa vulgarisation chez Le Caron et son exploitation en termes poétiques par Ronsard.

La moralisation de la fable est, pour Macrobe, une réponse savante et philosophique à sa condamnation par Platon et les philosophes. Elle permet une défense de la poésie. Celle-ci, en tant qu'elle est discours crypté mettant en jeu un sens caché qui demande un déchiffrement en termes allégoriques, se révèle capable d'embrasser tout le champ du savoir, comme une polymathie à visée encyclopédique. Par rapport aux commentaires grammaticaux, le commentaire de Macrobe est savant (jusqu’aux aspects les plus spéculatifs et les plus techniques portant sur la cosmologie) et moral ; il confirme d'une part les virtualités du Songe, un genre littéraire porteur plus que tout autre d'une capacité allégorique que ni Lemaire de Belges ni Ronsard ne négligèrent, et il offre d'autre part une méthode extraordinairement féconde, qui permet l'interprétation la plus riche de sens des œuvres poétiques. La lecture allégorique en clefs néoplatoniciennes que proposait Macrobe, par le système de correspondances qu'il impliquait, liant macrocosme et microcosme, réalité 
sensible et réalité intelligible, contribuait enfin à valoriser le poème, support d'une telle interprétation, en même temps qu'elle célébrait le poète comme un intermédiaire vers le cosmos et le divin. Macrobe semble avoir été à l'origine de la conception que Ficin développe de la fureur, qui confère au poète un statut d'élu, en lui reconnaissant le pouvoir d'entendre la musique des sphères célestes et le chant des Muses, et de les reproduire en écho dans ses propres hymnes, comme de recréer une nature idéale et parfaite à partir de l'imitation de la nature. Si en tant que grammairien Macrobe a joué un rôle déterminant à la Renaissance dans la compréhension littéraire et rhétorique de la poésie antique et dans le culte porté par l'Europe lettrée à Virgile, qui offrait la synthèse de tous les styles en une varietas supérieurement harmonieuse, en tant que philosophe, il est à l'origine de la célébration de la poésie comme théologie poétique, égale en dignité à la théologie dogmatique, à la philosophie et au droit, et partant, de la création ou plutôt de la redécouverte, transmise par l'intermédiaire de Ficin puis de Vida, d'une figure nouvelle, celle du poète inspiré, rattaché par une chaîne d'or à une longue tradition apollinienne et orphique, et qui, au XVI ${ }^{e}$ siècle, affirme son magistère en langue vulgaire, à travers l'allégorie du Parnasse.

JeAn Balsamo, Université de Reims

Ut philosophia poesis. Questions philosophiques dans l'ouvre de Dante, Pétrarque et Boccace.

Textes réunis par Joël Biard et Fosca Mariani Zini.

Paris : Vrin, De Pétrarque à Descartes, 2008, 285 p.

Six études portant sur Dante, trois sur Pétrarque et deux sur Boccace composent ce volume qui a pour but de comprendre pourquoi et comment la poésie choisit de se référer à la philosophie.

Le premier article, celui d'Irène Rosier-Catach ("'Il n’a été donné qu’à l'homme de parler' : Dante, les anges et les animaux »), étudie avec précision pourquoi Dante, au début du De vulgari eloquentia, attribue à l'homme seul le "parler»(locutio) qui laisse de côté la communication des anges, toute spirituelle, et celle des animaux, toute instinctive. Dante choisit un raisonnement fondé sur Aristote (commenté certes par saint Thomas), mais orienté par l'idée qu'avant la correction grammaticale compte l'adéquation du 